\title{
Inhibition of NADPH Oxidase Activation by Apocynin Rescues Seizure-Induced Reduction of Adult Hippocampal Neurogenesis
}

\author{
Song Hee Lee ${ }^{1}$, Bo Young Choi ${ }^{1}$, A Ra Kho ${ }^{1}$, Jeong Hyun Jeong ${ }^{1}$, Dae Ki Hong ${ }^{1}$, \\ Dong Hyeon Kang ${ }^{1}$, Beom Seok Kang ${ }^{1}$, Hong Ki Song ${ }^{2,3, *}$, Hui Chul Choi ${ }^{2,3, *}$ and \\ Sang Won Suh $1,3, *$ \\ 1 Department of Physiology, College of Medicine, Hallym University, Chuncheon 24252, Korea; \\ sshlee@hallym.ac.kr (S.H.L.); bychoi@hallym.ac.kr (B.Y.C.); rnlduadkfk136@hallym.ac.kr (A.R.K.); \\ jd1422@hanmail.net (J.H.J.); zxnm01220@gmail.com (D.K.H.); ehdgus2728@naver.com (D.H.K.); \\ ttiger1993@gmail.com (B.S.K.) \\ 2 College of Medicine, Neurology, Hallym University, Chuncheon 24252, Korea \\ 3 Hallym Institute of Epilepsy Research, Chuncheon 24252, Korea \\ * Correspondence: hksong0@hanmail.net (H.K.S.); dohchi@naver.com (H.C.C.); swsuh@hallym.ac.kr (S.W.S.); \\ Tel.: +82-10-8573-6364 (S.W.S.); Fax: +82-33-248-2580 (S.W.S.)
}

Received: 13 September 2018; Accepted: 2 October 2018; Published: 9 October 2018

\begin{abstract}
Apocynin, also known as acetovanillone, is a natural organic compound structurally related to vanillin. Apocynin is known to be an inhibitor of NADPH (Nicotinamide adenine dinucleotide phosphate) oxidase activity and is highly effective in suppressing the production of superoxide. The neuroprotective effects of apocynin have been investigated in numerous brain injury settings, such as stroke, traumatic brain injury (TBI), and epilepsy. Our lab has demonstrated that TBI or seizure-induced oxidative injury and neuronal death were reduced by apocynin treatment. Several studies have also demonstrated that neuroblast production is transiently increased in the hippocampus after seizures. Here, we provide evidence confirming the hypothesis that long-term treatment with apocynin may enhance newly generated hippocampal neuronal survival by reduction of superoxide production after seizures. A seizure was induced by pilocarpine [( $25 \mathrm{mg} / \mathrm{kg}$ intraperitoneal (i.p.)] injection. Apocynin was continuously injected for 4 weeks after seizures (once per day) into the intraperitoneal space. We evaluated neuronal nuclear antigen (NeuN), bromodeoxyuridine (BrdU), and doublecortin (DCX) immunostaining to determine whether treatment with apocynin increased neuronal survival and neurogenesis in the hippocampus after seizures. The present study indicates that long-term treatment of apocynin increased the number of $\mathrm{NeuN}^{+}$and $\mathrm{DCX}^{+}$cells in the hippocampus after seizures. Therefore, this study suggests that apocynin treatment increased neuronal survival and neuroblast production by reduction of hippocampal oxidative injury after seizures.
\end{abstract}

Keywords: epilepsy; pilocarpine; neuron death; NADPH-oxidase inhibitor; oxidative stress; neurogenesis

\section{Introduction}

An epileptic seizure is a transient or recurrent display of related symptoms due to abnormal excessive or synchronous neuronal activity in the brain. Epilepsy is now termed a disease, rather than a disorder. When epilepsy occurs, there is a high probability that it may develop into recurring epilepsy (chronic epilepsy) [1-3]. Epilepsy leads to neuronal death in the hippocampus, which manifests as cognitive and behavioral impairments. Following periods of severe epileptic seizures, there is a 
dramatic increase in hippocampal cellular proliferation and neurogenesis. Neurogenesis is the process by which neurons are created from neural stem cells, and occurs continuously in certain parts of the adult brain, especially in the subgranular zone (SGZ) of the hippocampal dentate gyrus (DG). Most of the newly born neurons are produced in the SGZ and die shortly after they are born. A smaller fraction are functionally integrated with surrounding brain areas [4]. Neurogenesis is highly influenced by learning and memory, emotions, stress, and other conditions [5]. Neurogenesis in the DG is known to be associated with hippocampal function for learning and memory [6,7]. In particular, neurogenesis occurs in response to brain damage, and studies on neurogenesis have been extensively conducted in various animal models. However, the topic is not without controversy and for decades the effect of seizures on neuron death and neurogenesis has been debated [8-11].

Epileptic seizure is known to increase hippocampal neurogenesis [12,13]. However, recurrent and continuous seizures can lead to an ongoing increase in oxidative stress and a constant suppression of the hippocampal neurogenic process [14-16]. In addition, the reduction of neural development in the hippocampus is one of the primary causes of hippocampus-dependent learning and cognitive memory impairment observed after hippocampal damage due to epilepsy or other diseases [15]. After seizures, oxidative stress is increased, and the perturbation of redox balance in the hippocampus can reduce neurogenesis [14].

Apocynin is structurally related to natural vanillin, which is extracted from various plants and has been studied for its various pharmacological properties $[17,18]$. Apocynin is also known to inhibit NADPH (Nicotinamide adenine dinucleotide phosphate) oxidase by inhibiting p47phox subunit translocation from the cytoplasm to the plasma membrane area $[19,20]$. In many other studies, apocynin has been shown to successfully reduce reactive oxygen species (ROS) and reduce cell death [21-23]. Our previous studies demonstrated that pilocarpine-induced seizures increased the generation of ROS in hippocampal neurons. Furthermore, treatment with apocynin after seizures reduced neurodegeneration in other studies [24,25]. Our previous study also demonstrated that production of ROS by NADPH oxidase activation induced neuronal cell death after traumatic brain injury or hypoglycemia [21,22,26-28]. Although we have demonstrated that effects of NADPH oxidase activation in neuronal death after hypoglycemia [26], traumatic brain injury [22], and epilepsy [21], the efficacy of inhibition of NADPH oxidase activation by apocynin treatment in seizure-induced neurogenesis has not been demonstrated. We hypothesized that, if ROS are constantly produced after a seizure, the rate of newly proliferated neuronal survival is reduced as a function of the ROS production [29-31]. In the present study, we found that the survival rate and neurogenesis of hippocampal cells after seizure induction was increased via inhibition of NADPH oxidase. We found that neurogenesis is increased in the early stages of a seizure, but declines after 4 weeks. However, in rats treated with apocynin for 4 weeks after seizures, neuronal death was reduced, and the survival rate of early proliferated cells increased.

Thus, the present study suggests that treatment with apocynin may have a high therapeutic potential for increasing neurogenesis, as well as reducing seizure-induced neuronal death through reduction of ROS production.

\section{Results}

\subsection{Apocynin Treatment Reduces Seizure-Induced Hippocampal Neuronal Death}

To determine whether apocynin therapy is effective in neuroprotection, we measured the number of live neurons by staining with anti-neuron nuclear antigen (NeuN) one week after the seizure. The number of $\mathrm{NeuN}^{+}$cells was decreased in the Cornu Ammonis 1 and 3 (CA1 and CA3), hilus, and subiculum (Sub) regions of the hippocampus after the seizure (Figure 1A). In the CA1, CA3, hilus, and subiculum areas, the number of $\mathrm{NeuN}^{+}$neurons in the seizure-vehicle-treated group decreased, when compared to the sham-vehicle group. Apocynin treatment for 1 week significantly reduced neuronal death in each region compared to the vehicle-treated seizure group. In the CA3 region, 
neuronal death was less in the seizure-apocynin group, but this was not significantly different after analysis. This result shows that the number of viable neurons is higher in the apocynin-treated group and that apocynin-treatment reduces seizure-induced neuronal death.

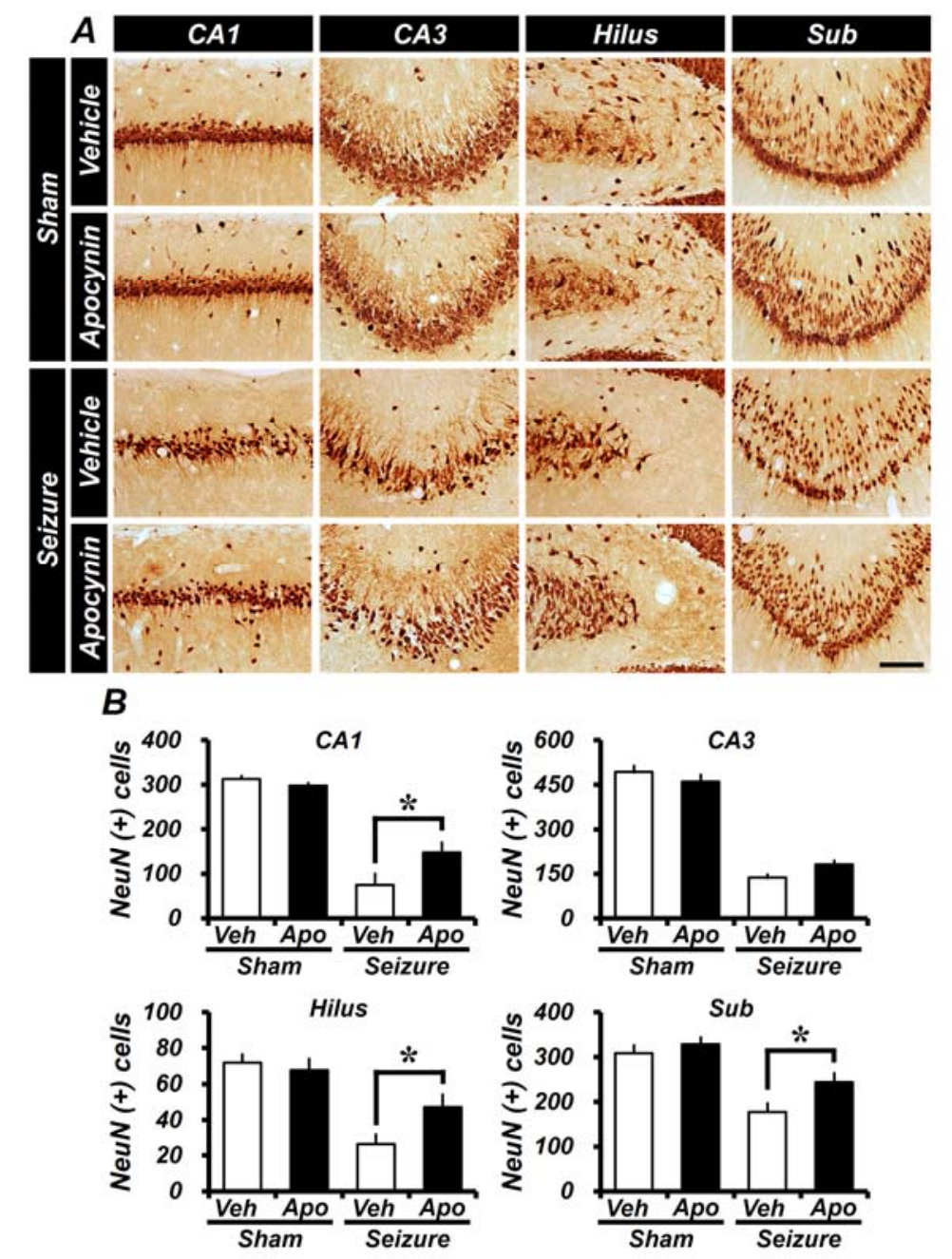

Figure 1. Treatment with apocynin for a week reduces seizure-induced neuronal cell death. Anti-neuron nuclear antigen (NeuN) staining was performed to identify live neurons. (A) A micrograph showing live neurons in the sham group, the seizure control group, and the apocynin treatment group. Apocynin treatment shows a substantially increased number of $\mathrm{NeuN}^{+}$cells in the hippocampus of the seizure group. Scale bar $=100 \mu \mathrm{m}$. (B) The graph shows the average number of live neurons ( $\mathrm{NeuN}^{+}$cells) in the hippocampus after seizure compared to the apocynin treatment group. Data: mean \pm standard error of the mean (S.E.M.), $n=5-6$ from each group. ${ }^{*} p<0.05$. CA3 is not statistically significant. Veh = Vehicle; Apo = Apocynin; CA1 and CA3 = Cornu Ammonis 1 and 3 of the hippocampus area; Sub $=$ Subiculum.

\subsection{Apocynin Treatment for One Week Reduces Oxidative Damage after Seizure in the Hippocampus}

To assess the degree of oxidative damage in the hippocampus, apocynin was administered for one week and then $4 \mathrm{HNE}$ (4-hydroxy-2-nonenal) antibody was used for immunohistochemical staining. In the sham group, there was almost no $4 \mathrm{HNE}$ fluorescence staining in both saline injected vehicle and in apocynin injected group. However, in the hippocampus of the pilocarpine-induced seizure group, the intensity of $4 \mathrm{HNE}$ fluorescence was increased when compared to sham group. As quantified in Figure 2B, the intensity of $4 \mathrm{HNE}$ fluorescence intensity was increased in the hippocampal CA1, CA3, hilus and subiculum compared to the sham group. The $4 \mathrm{HNE}$ fluorescence intensity was lower in the apocynin group than in the vehicle group. The intensity of the $4 \mathrm{HNE}$ signal in CA1, CA3, hilus and 
subiculum was $40 \pm 2.6 \%, 47 \pm 3.5 \%, 48 \pm 7.5 \%$ and $46 \pm 3.6 \%$ lower, in the apocynin treated group compared to the seizure-vehicle group (Figure 2).

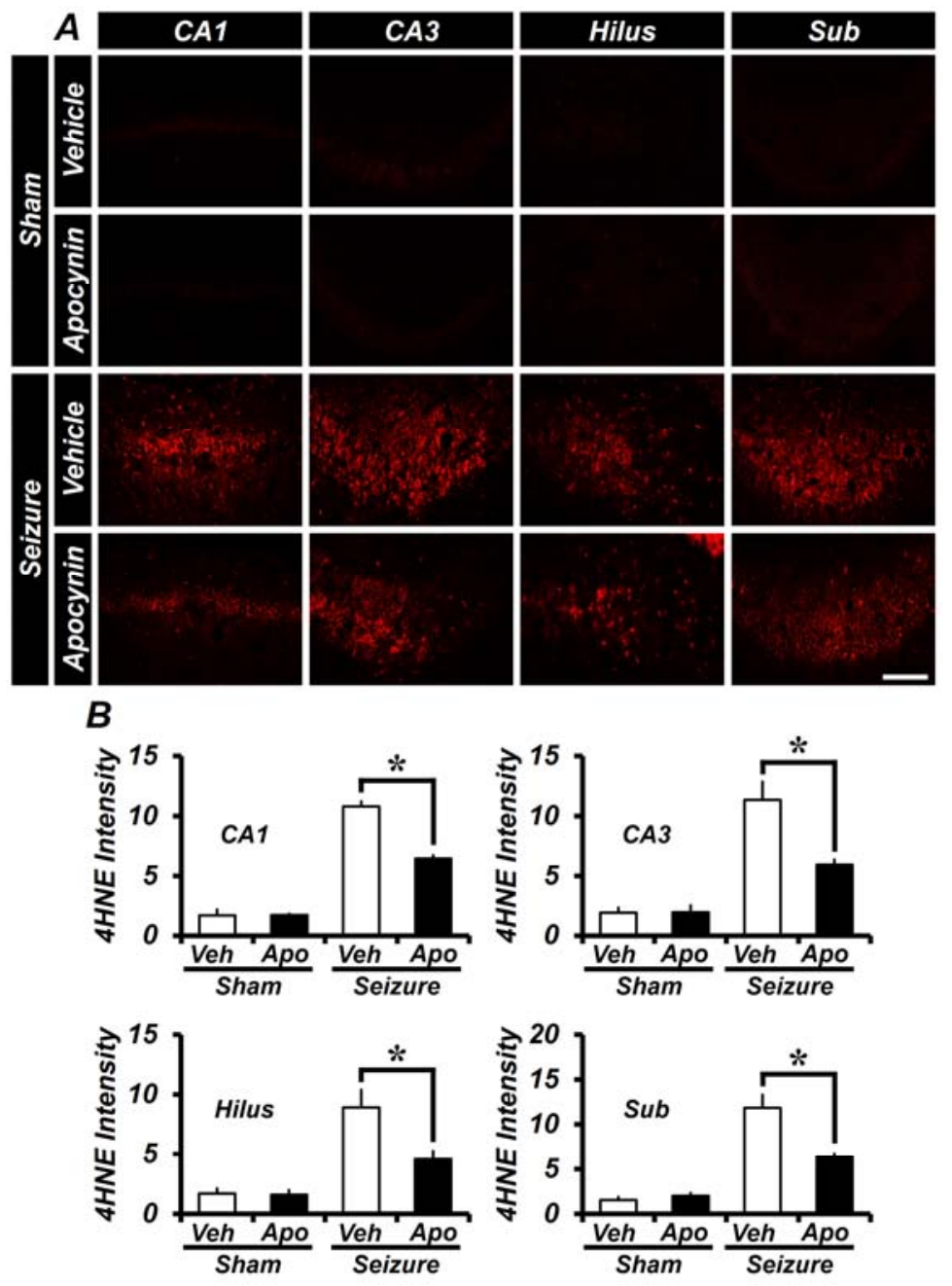

Figure 2. Apocynin administration reduces oxidative injury after seizure. Oxidative stress was observed by anti-4-hydroxynonenal (4HNE) immunostaining after one week of seizure. (A) The intensity of $4 \mathrm{HNE}$ was increased in the seizure-vehicle group when compared to the sham group. In the seizure-apocynin treated group, the $4 \mathrm{HNE}$ intensity was lower than the vehicle treated group. Scale bar $=100 \mu \mathrm{m}$. (B) The bar graph shows the $4 \mathrm{HNE}$ fluorescence intensity of the hippocampus shown in (A). Data: Mean \pm S.E.M., $n=5-6$ from each group, ${ }^{*} p<0.05$.

\subsection{Apocynin Treatment for One Week Does Not Affect the Generation of Progenitor Cells and Production of} Neuroblast in the Hippocampus after Seizure

Immunohistochemical staining with 5-bromo-2'-deoxyuridine (BrdU) and doublecortin (DCX) antibody was performed to determine whether immediate 1-week apocynin treatment affected the production of cellular proliferation and recognizes neuroblasts or immature neurons after a seizure. BrdU was administered twice daily $(30 \mathrm{mg} / \mathrm{kg}$, i.p.) to rats 4 days before sacrifice following a pilocarpine-induced seizure. We quantified $\mathrm{BrdU}^{+}$cells showing proliferating cells after a seizure. After one week of pilocarpine-induced seizures, the number of $\mathrm{BrdU}^{+}$cells were significantly increased in the subgranular zone (SGZ) of the vehicle-treated and apocynin-treated groups, respectively, compared to the sham-vehicle-treated group. However, the number of $\mathrm{BrdU}^{+}$cells in the apocynin-treated group was similar between the two groups when compared to the vehicle-treated group after one week of seizures. DCX immunostaining is effective in identifying and quantifying newly born neuroblasts in the adult DG. Detection of immunologic markers using DCX antibodies showed neuroblasts in the SGZ 
in the DG region. One-week treatment with apocynin increased neuroblasts in both seizure groups compared to the sham-vehicle-treatment group, but did not show any difference in neuroblastogenesis in the SGZ of the DG when compared with seizure-vehicle-treated and apocynin-treated groups. These results indicate that treatment with apocynin for one week after seizure has no effect on seizure-induced cellular proliferation and production of neuroblasts after seizure (Figure 3).
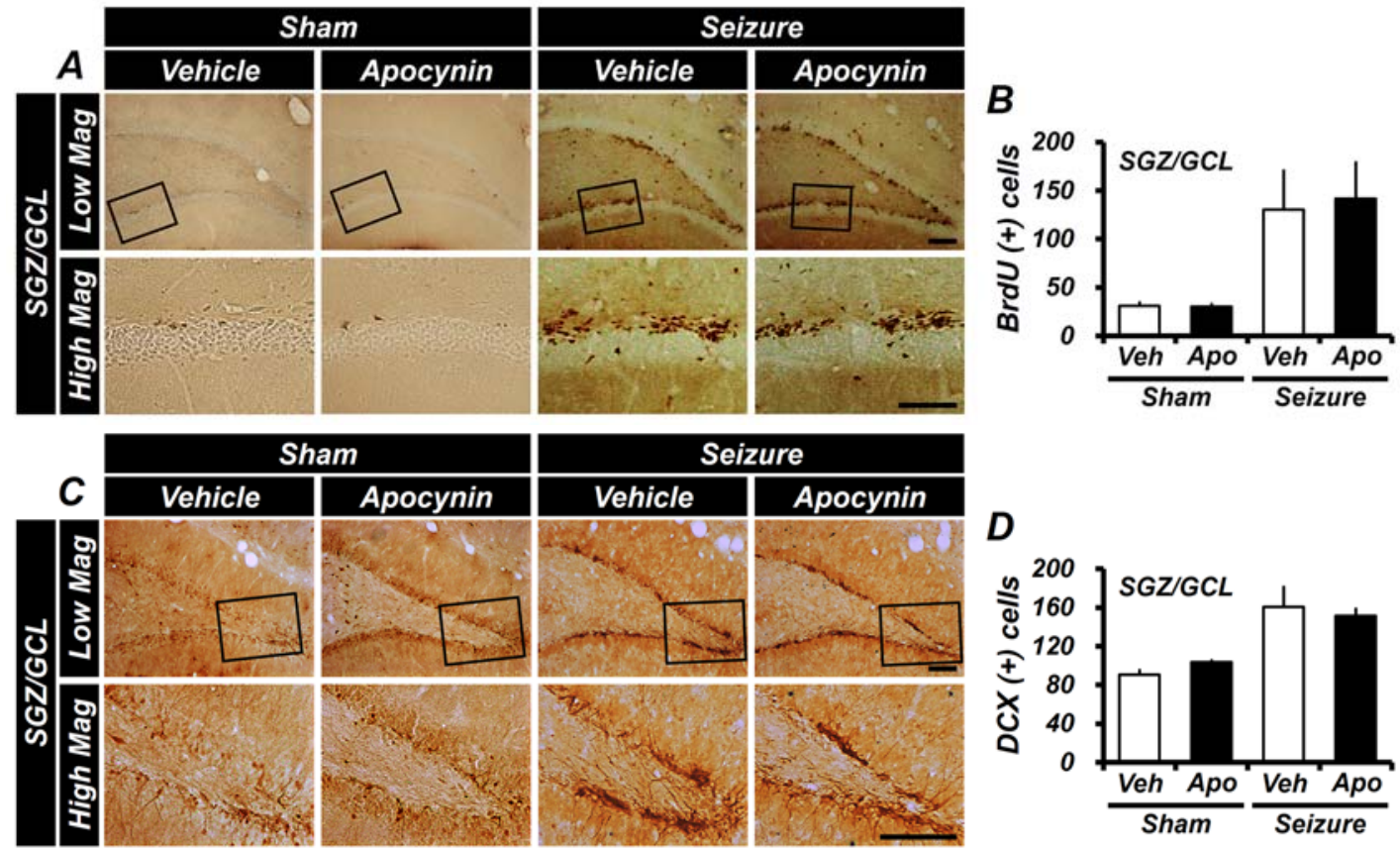

Figure 3. Treatment with apocynin for one week does not affect cell proliferation and neuroblast production after seizures. (A) Representative immunohistochemical staining with 5-bromo-2'-deoxyuridine (BrdU) in the subgranular zone (SGZ) of dentate gyrus (DG). A cluster of proliferation cells labeled with BrdU in the SGZ immediately following a seizure is shown. BrdU ${ }^{+}$ cells are increased in the SGZ of the DG both in the vehicle group and in the apocynin group after 1 week of seizures. Boxed areas are higher magnification (bottom row). Scale bar $=100 \mu \mathrm{m}$. (B) The bar graph shows the quantification of $\mathrm{BrdU}^{+}$cells from the SGZ of the DG. The seizure group, compared with the sham group, showed a greater increase in $\mathrm{BrdU}^{+}$cells. There was no significant difference between the seizure-vehicle- and apocynin-treated groups. (C) This figure shows the doublecortin (DCX)-immunoreactive cells in the SGZ of the DG and shows the neuroblast production by enlarging the DG and box area. In the DG of the hippocampal area where a seizure was induced by pilocarpine, more neuroblasts were observed than the uninjured hippocampus. Boxed areas are higher magnification (bottom row). Scale bar $=100 \mu \mathrm{m}$. (D) The bar graph is the result of counting $\mathrm{DCX}^{+}$cells stained in the DG of the hippocampus. $\mathrm{DCX}^{+}$cells increased more in seizure-induced rats compared with the sham group. There was no significant difference between the vehicle- and apocynin-treated groups. Data: Mean \pm S.E.M., $n=5-6$ from each group. Low Mag, low magnification; High Mag, high magnification.

\subsection{Seizure-Induced Hippocampal Neuronal Death Is Reduced by Apocynin Treatment for Four Weeks}

Neuronal cell death was assessed by immunohistochemical staining using NeuN, and the effect of apocynin on neuronal cell death was confirmed in hippocampal CA1, CA3, hilus, and subiculum areas. Four weeks after pilocarpine-induced seizures, $\mathrm{NeuN}^{+}$neurons in each area of the hippocampus were found to have a reduced number of live neurons in both seizure-induced groups compared to the sham-vehicle group. The number of live neurons in the seizure-vehicle-treated group in CA1, CA3, the DG, and the subiculum was reduced compared to the sham-vehicle-treated group. However, in animals treated with apocynin, the number of NeuN positive cells was higher than that of the seizure-vehicle-treated group than the seizure group. These results show that apocynin treatment, 
even given 4 weeks after the induction of seizures, reduces neuronal damage that occurs in this setting (Figure 4).

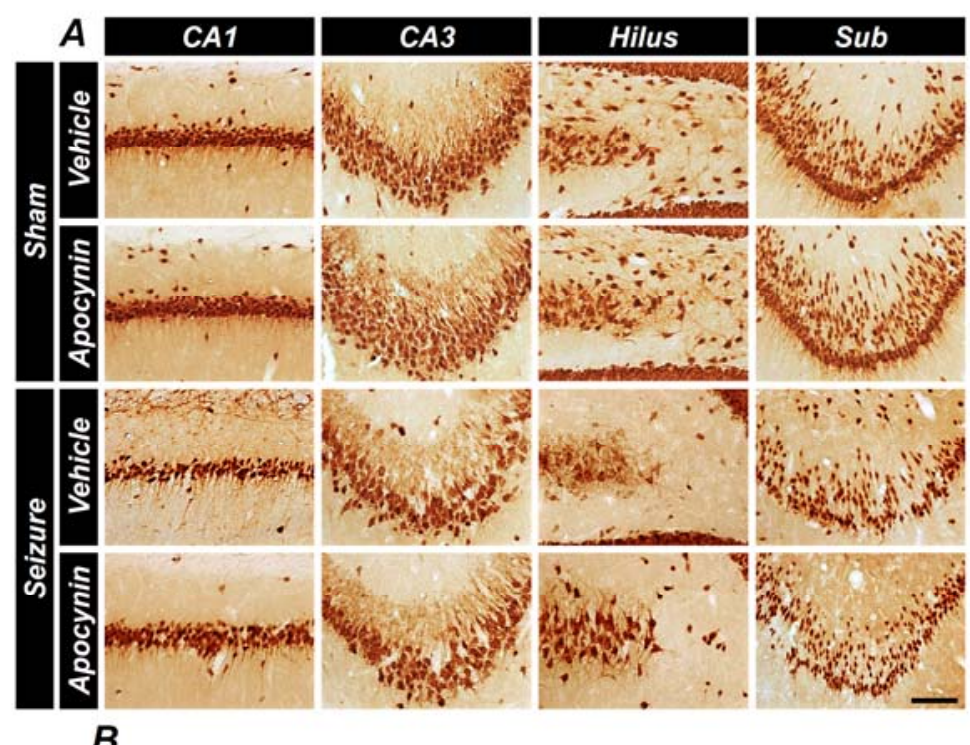

\section{$B$}
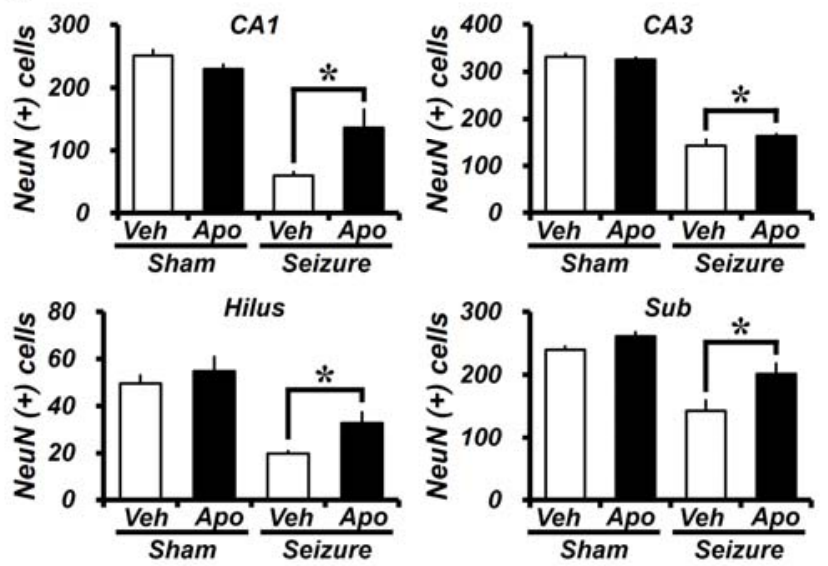

Figure 4. Apocynin treatment reduces neuronal cell loss induced by seizures. (A) Live neurons or mature neurons in the hippocampus were identified by NeuN immunohistochemical staining. Scale bar $=100 \mu \mathrm{m}$. (B) Bar graph showing the number of $\mathrm{NeuN}^{+}$neurons in the hippocampus treated with apocynin for 4 weeks after seizures. Among the two groups receiving seizures, the apocynin-treated group had a greater number of neurons when $\mathrm{NeuN}^{+}$cells were compared to the vehicle-treated group. Seizure-induced $\mathrm{NeuN}^{+}$neuron loss was prevented by apocynin treatment in the hippocampus each region compared to the vehicle treatment. Data: Mean \pm S.E.M., $n=5-8$ from each group, ${ }^{*} p<0.05$.

\subsection{Treatment of Apocynin for 4 Weeks after Seizures Reduces Oxidative Damage in the Hippocampus}

A 4HNE (4-hydroxy-2-nonenal) antibody was used to evaluate the occurrence of oxidative damage in the hippocampus of the brain when apocynin was administered for 4 weeks after seizure. Figure 5B shows that the intensity of $4 \mathrm{HNE}$ in the seizure-vehicle group increased in the hippocampal region, when compared to the sham-vehicle group. The seizure apocynin-treated group had a lower $4 \mathrm{HNE}$ intensity than the vehicle-treated group. CA1, CA3, hilus and subiculum in the apocynin-treated group were reduced by $31 \pm 8.7 \%, 36 \pm 4.8 \%, 30 \pm 8.1 \%$ and $40 \pm 6.8 \%$, compared to the vehicle-treatment group (Figure 5). 

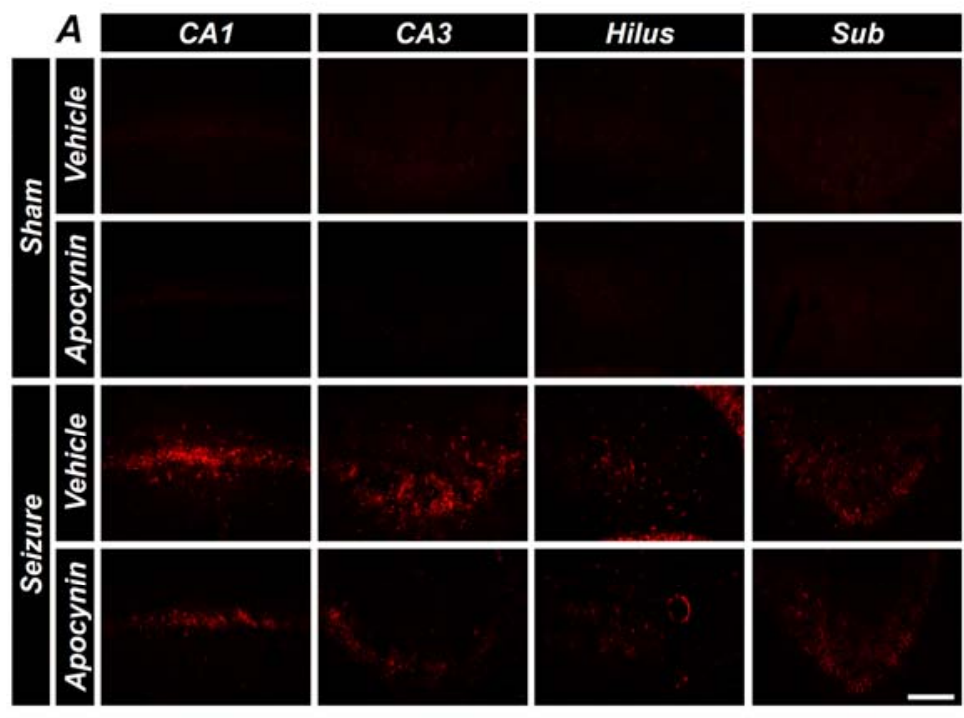

\section{B}
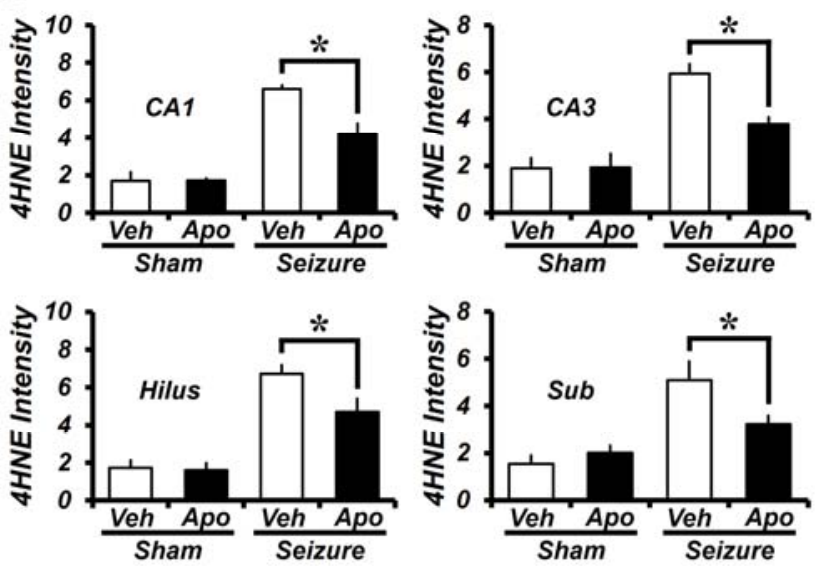

Figure 5. Apocynin treatment reduces $4 \mathrm{HNE}$ levels in the hippocampus at 4 weeks after seizures. (A) A microscopic image showing oxidative injury with $4 \mathrm{HNE}$ at each region of the hippocampus. The intensity of $4 \mathrm{HNE}$ was increased in the seizure group when compared to the sham-vehicle group. The $4 \mathrm{HEN}$ intensity was reduced in the seizure-apocynin treatment group when compared to the seizure-vehicle group. Scale bar $=100 \mu \mathrm{m}$. (B) The bar graph shows the quantification of the levels of $4 \mathrm{HNE}$ in each region of the hippocampus. Data: Mean \pm S.E.M., $n=5-8$ from each group, ${ }^{*} p<0.05$.

\subsection{Apocynin Treatment Increases the Survival Rate of Newly Born Neurons and Newly Generated Neurons}

Cellular proliferation is increased in the early stages after a seizure, but survival of proliferated cells is not maintained 4 weeks post-insult $[4,16]$. The BrdU experiments at 1 and 4 weeks show that the proliferating cells, which were increased 1 week after seizures, were decreased after 4 weeks. BrdU staining was performed to confirm the survival of initially labeled $\mathrm{BrdU}^{+}$cells after apocynin treatment for 4 weeks after seizures. After 4 weeks of seizure induction, it was confirmed that the number of BrdU-labeled cells at the early time of seizures decreased. Apocynin was injected for 4 weeks after seizures, and the survival count of the initially generated cells was found to be higher than in the vehicle-treated group. Furthermore, the survival of proliferating cells after a seizure was increased by continuous apocynin treatment more than the vehicle treatment (Figure 6A,B). Neuronal proliferation increased in the early stages after seizures. Analysis of neuroblasts 4 weeks after induction of seizures with pilocarpine showed the following results. Analysis of the number of neuroblasts revealed that the number of neuroblasts in the chronic interstitial hippocampus was significantly reduced. The density of newborn neurons in the SGZ was considerably greater after seizure-induced cell loss. At 4 weeks of pilocarpine-induced seizures, the neuroblast production in the vehicle-treated group was decreased 
compared to the initial vehicle-treated group. However, neuroblast production in the apocynin-treated group was higher than the vehicle-treated group (Figure $6 \mathrm{C}, \mathrm{D}$ ). BrdU and NeuN double staining was performed to determine whether the proliferating cells were differentiated into mature neurons. It was confirmed that the number of $\mathrm{BrdU}^{+} \mathrm{NeuN}^{+}$cells labeled 4 weeks after seizures was decreased. Apocynin was injected for 4 weeks after seizures and the number of differentiated mature neurons among surviving cells was higher than in the vehicle group (Figure 6E,F).

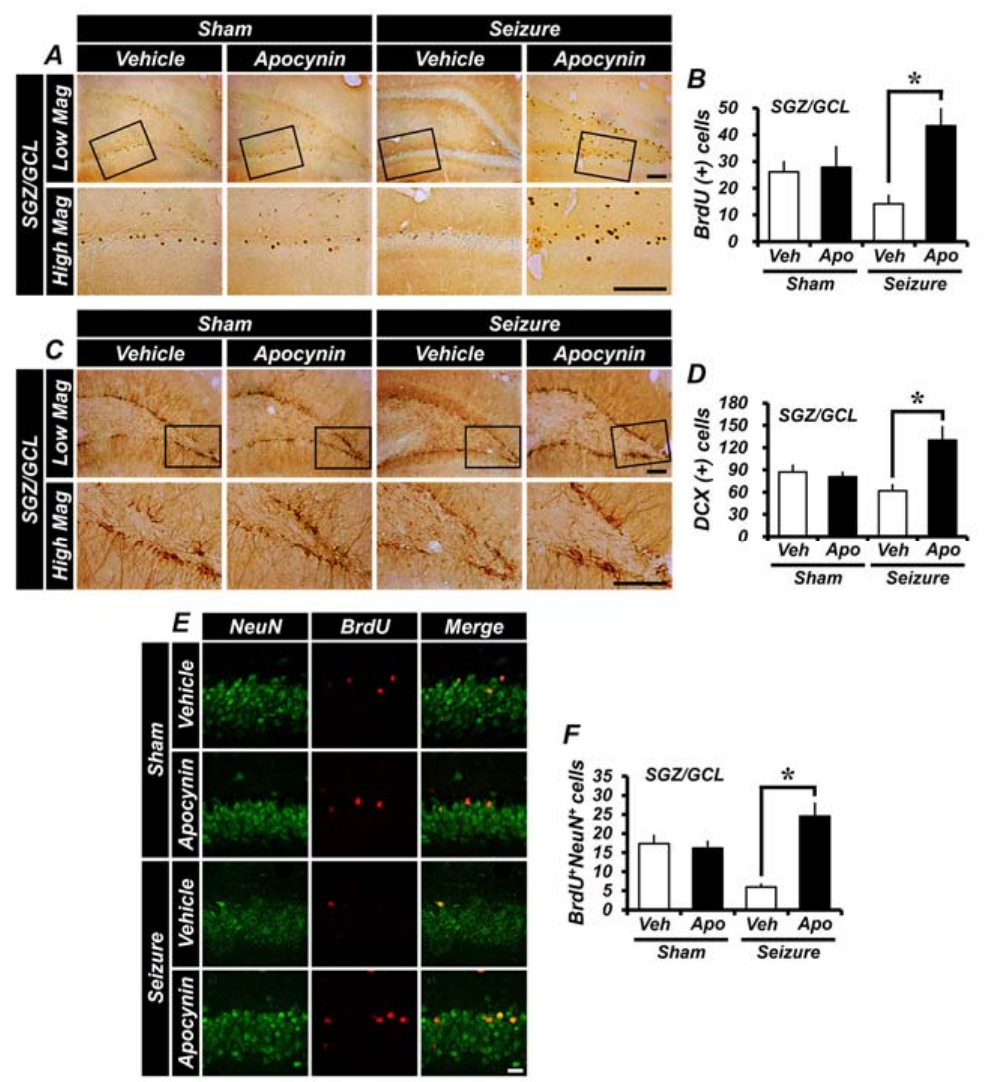

Figure 6. Apocynin treatment increases the survival rate of newly generated cells and neuroblast production in the SGZ of the DG. The survival rate of newly generated cells was assessed by BrdU staining. Long-term survival of newly proliferated cells was increased by apocynin treatment. Differentiation of newly proliferated cells into mature neurons was increased by apocynin treatment. (A) BrdU ${ }^{+}$cells are shown in the SGZ of the DG. Four weeks after injecting BrdU in an adult (8-9 weeks old), BrdU-labeled cells can be seen in the granule cell layer. After 4 weeks of inducing seizures, the number of cells labeled with BrdU decreased. The apocynin-treated group had a decreased number of BrdU-labeled cells after seizures, but still greater than the vehicle-treated group. Boxed areas are higher magnification (bottom row). Scale bar $=100 \mu \mathrm{m}$. (B) The graph shows BrdU-labeled cells quantified in the hippocampus. The number of BrdU ${ }^{+}$cells (survived for 4 weeks) was significantly higher in apocynin-treated rats than in vehicle-treated rats. (C) Immunohistochemical staining of this figure confirmed the presence of neuroblasts. In the seizure model, when the $\mathrm{DCX}^{+}$cells were identified in the SGZ of the DG 4 weeks after apocynin treatment, the number of cells was significantly increased compared with the vehicle-treated group. Boxed areas are higher magnification (bottom row). Scale bar $=100 \mu \mathrm{m}$. (D) The graph is the mean value obtained from five individual sections and represents the number of $\mathrm{DCX}^{+}$cells in the SGZ of the DG. (E) $\mathrm{BrdU}^{+} \mathrm{NeuN}^{+}$cells are shown in the hippocampal DG region. BrdU ${ }^{+} \mathrm{NeuN}^{+}$cells were identified in the granule cell layer at 4 weeks after BrdU injection. Scale bar $=100 \mu \mathrm{m}$. (F) The graph shows BrdU/NeuN labeled cells quantified in hippocampus. The number of $\mathrm{BrdU}^{+} \mathrm{NeuN}^{+}$cells (survival for 4 weeks) was significantly higher than in the vehicle-treated group in the apocynin-treated group. Data: Mean \pm S.E.M., $n=5-8$ from each group, ${ }^{*} p<0.05$. Low Mag, low magnification; High Mag, high magnification. 


\section{Discussion}

Epilepsy is known to occur after primary damage due to cerebral infarction, stroke, or traumatic brain injury. Epilepsy is one of the most common neurological disorders, and it is reported that approximately 50 million people worldwide suffer from this disease. Repeated epilepsy can result in damage to the temporal lobe, especially the hippocampus [32]. Studies on epilepsy with animal models and evaluation of human brain tissue have revealed the cause of neuronal death including partial regression of CA1 and CA3 pyramidal neurons, dentate gyrus (DG), and subiculum (Sub) cells in the hippocampus, but the exact causes and treatments are yet to be identified.

The effect of short-term apocynin administration before and after the onset of epilepsy has been previously studied [21,23,24]. However, the long-term post-treatment effect of apocynin has not been tested. Neuron nuclear antigen (NeuN), bromodeoxyuridine (BrdU), and doublecortin (DCX) staining was performed to evaluate whether apocynin administration has a neuroprotective effect and can increase the survival rate of newly generated neurons. When hippocampal neurons were injured in a pilocarpine-induced epilepsy model, cellular proliferation measured by BrdU labeling was dramatically increased in the subgranular zone (SGZ) of the DG. The number of neuroblasts also increased two to three days after epilepsy.

NADPH oxidase has also been reported to be involved in the neurodegenerative process of NADPH oxidase-dependent ROS overproduction in other brain regions, such as the cerebellum, that are involved in the transactivation of CAMKII [33]. It is known that epilepsy and oxidative stress are closely interrelated. It is also known that an increase of free radicals and oxidative stress after epilepsy can lead to neuronal cell death, because the brain is known to be more vulnerable to damage by excess oxygen and oxidative stress than other tissues [34,35]. Apocynin is known to reduce oxidative damage through the inhibition of NADPH oxidase activation. To confirm whether apocynin has neuroprotective effects, we performed NeuN staining 1 week or 4 weeks after seizures. At each time point, the apocynin-treated group, compared with the vehicle-treated group, showed a higher number of viable neurons in the hippocampus. These results indicate that apocynin therapy either for 1 week or for 4 weeks reduces neuronal death and increases the number of surviving neurons after seizures.

Oxidative damage is involved in promoting apoptosis and is thought to play an important role as a causative agent of degenerative neuropathy [36,37]. In this study, we found that the oxidative damage of neurons increased after one and four weeks of seizures. Administration of apocynin reduced oxidative damage in seizure-induced rats. This finding confirms and extends the idea that apocynin reduces oxidative stress and thus reduces the death of newly generated cells after seizures, as well as existing neurons.

Recently, it has been demonstrated that NADPH oxidase-mediated ROS generation can contribute to the transactivation of TRKs (Tropomyosin receptor kinases), thus improving cell proliferation and viability [38]. Oxidative stress leads to the death of existing neurons, but also of newly generated cells, after seizures [39]. Since apocynin is known to prevent neuronal death, the present study was performed to test whether apocynin also prevented the death of newly generated cells in pilocarpine-induced epilepsy models. Previous studies have reported that neuronal development or new-cell generation is continuously decreased in the SGZ of the adult rat hippocampus after a seizure [30,31,40-42]. In the present study, we also found that the number of newly generated cells after a seizure is lower than in sham-group animals, which means either the new cellular proliferation rate is lowered, or that the newly generated cells were damaged. Cellular proliferation in the hippocampus was observed at one week after the pilocarpine-induced seizure, but there was no difference between the control and apocynin-treated groups. Thus, the present study was undertaken to evaluate the effect of apocynin treatment on the survival rate of neurons and newly formed cells in the early stages of epilepsy. We found that the survival rate of BrdU-labeled cells, when evaluated 4 weeks after the seizure, was only $20 \%$ in the SGZ of the DG. However, at 4 weeks, the apocynin-treated group showed that the survival rate of BrdU-labeled cells was $40 \%$. This result shows that apocynin 
treatment increases the survival rate of early-proliferated cells. The present study also shows that immediate administration of apocynin after seizures, for 1 week, was effective in decreasing neuronal cellular death, but administration of apocynin showed no beneficial effects on neuroblast generation. The number of neuroblasts after seizures was similar in both apocynin- and vehicle-treated rats one week after the seizure. However, we found that administration of apocynin for 4 weeks showed an increased number of neuroblasts in the hippocampus compared to the vehicle-treated group. It has been found that apocynin reduces oxidative injury and reduces apoptosis of newly generated neurons, leading to oxidative injury and to the death of newly generated cells, as well as existing neurons. This study shows that damage caused by oxidative injury after seizures was reduced by apocynin, thereby reducing the death of generated cells, as well as existing neurons, after seizures. This result demonstrates that inhibition of ROS production by apocynin increases neuroblast survival after seizure.

Two key results of the present study are as follows: (1) Apocynin increases the survival rate of newly formed cells; (2) apocynin increases the number of neuroblasts in the hippocampus after pilocarpine-induced seizures.

\section{Materials and Methods}

\subsection{Ethics Statement}

This study was performed in accordance with the guidelines of the Laboratory Animal Care and Use Guide issued by the National Institutes of Health (NIH). Animal studies are consistent with the requirements of the Hallym University Animal Care Committee (Protocol \# Hallym 2016-70, approval date: 9 February 2016). Every effort, such as the use of isoflurane anesthesia, was made to minimize the pain of animals sacrificed.

\subsection{Experimental Animals}

In this study, 8-week-old Sprague-Dawley male rats (250-300 g, Daehanbiolink (DBL) Co., Chungcheongbuk-do, Korea) were used. Subjects were maintained at a constant room temperature $\left(22 \pm 2{ }^{\circ} \mathrm{C}\right)$ and humidity $(55 \pm 5 \%)$, one per cage, and the room lighting was automatically turned on and off at every $12 \mathrm{~h}$ (6:00 a.m. and 6:00 p.m.). The animals were divided into four groups for Week 1 (sham-vehicle $n=5$; sham-apocynin $n=5$; seizure-vehicle $n=6$; seizure-apocynin $n=6$ ) and Week 4 (sham-vehicle $n=5$; sham-apocynin $n=5$; seizure-vehicle $n=6$; seizure-apocynin $n=8$ ) groups.

\subsection{Experimental Procedures}

Lithium chloride ( $\mathrm{LiCl}, 127 \mathrm{mg} / \mathrm{kg}$, i.p., Sigma-Aldrich Co., St. Louis, MO, USA) was administered $19 \mathrm{~h}$ before the injection of pilocarpine and induced epileptic seizures with pilocarpine $(25 \mathrm{mg} / \mathrm{kg}$, i.p., Sigma-Aldrich Co., St. Louis, MO, USA) [43]. Scopolamine ( $2 \mathrm{mg} / \mathrm{kg}$, i.p., Sigma-Aldrich Co., St. Louis, MO, USA) was injected $30 \mathrm{~min}$ before the induction of seizures with pilocarpine. Seizures are generally known to occur 20-30 min after injection of pilocarpine [44]. Pilocarpine was administered only once at the day of the experiment in which the epilepsy is induced. Animals were housed one per cage for behavioral observations of individual seizures. According to the Racine stage, described by Racine, five symptoms are described when a seizure occurs (1. Mouth and facial movements; 2 . Head nodding; 3 . Forelimb clonus; 4 . Rearing with forelimb clonus; and 5. Rearing and falling with forelimb clonus). We judged that a complete seizure has occurred as soon as the fifth symptom is observed [45]. The seizure was maintained after the seizure was complete, and diazepam (Valium, $10 \mathrm{mg} / \mathrm{kg}$, i.p., Hoffman la Roche, Neuilly sur-Seine, Roche Co., Basel, Switzerland) was injected $2 \mathrm{~h}$ later. Diazepam ( $2 \mathrm{mg} / \mathrm{kg}$, i.p.) was injected to stop residual seizure activity when recurrent severe seizures were observed after diazepam treatment [21,46] (Figure 7A). Apocynin and 5-bromo-2-deoxyuridine (BrdU) injections were divided into four groups of animals: Sham-dimethyl sulfoxide (DMSO) (Sigma-Aldrich Co., St. Louis, MO, USA, 0.01 mL/kg, pH 7-7.4, i.p.), sham-apocynin (Sigma-Aldrich Co., St. Louis, $\mathrm{MO}$, USA, $30 \mathrm{mg} / \mathrm{kg}$, i.p.), seizure-DMSO, and seizure-apocynin. Animals were injected with apocynin 
once a day for 1 week and 4 weeks after pilocarpine-induced seizures. Apocynin was injected $2 \mathrm{~h}$ after the seizure, and 2 injections $24 \mathrm{~h}$ after the seizure. After one week of seizures, BrdU ( $50 \mathrm{mg} / \mathrm{kg}$, i.p., Sigma-Aldrich Co., St. Louis, MO, USA) was administered into intraperitoneal space twice a day, from 4 days before sacrifice, for 4 days to observe cell proliferation. In addition, to investigate the survival of the proliferated cells, animals were sacrificed 4 weeks after seizure (Figure 7B,C).

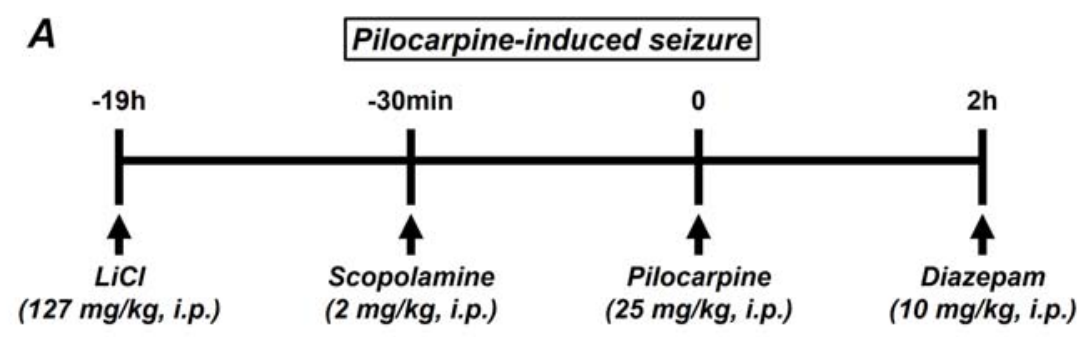

B Proliferation following pilocarpine-induced seizure

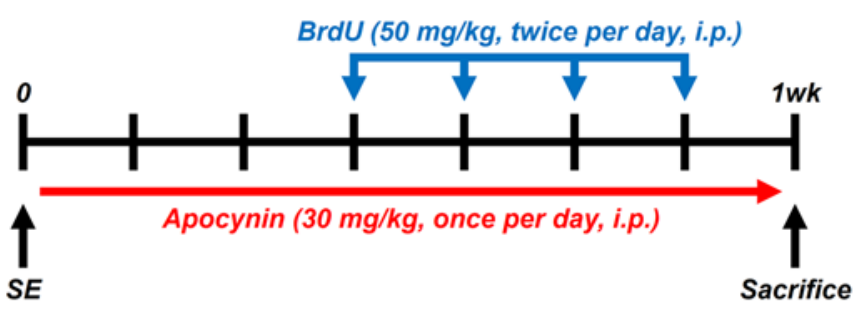

C Survival of newborn cells

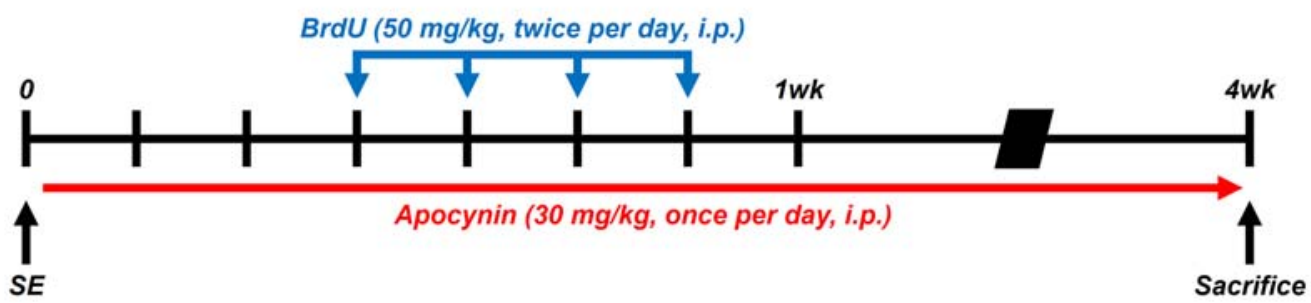

Figure 7. (A) Schematic of pilocarpine-induced seizure. Lithium chloride ( $\mathrm{LiCl} 127 \mathrm{mg} / \mathrm{kg}$, i.p.) was administered $19 \mathrm{~h}$ before induction of seizure by pilocarpine. Scopolamine $(2 \mathrm{mg} / \mathrm{kg}$, i.p.) was injected $30 \mathrm{~min}$ before administered pilocarpine. Pilocarpine was injected at $25 \mathrm{mg} / \mathrm{kg}$, i.p. After a seizure with pilocarpine, diazepam (10 mg/kg, i.p.) was administered $120 \mathrm{~min}$ later to lower the seizure. (B,C) Schematic of key experiments performed in this study. Proliferation cells were confirmed in rats after pilocarpine-induced seizures. BrdU ${ }^{+}$cells were quantified 1 or 4 weeks after the seizure. SE, status epilepticus.

\subsection{Brain Sample Preparation}

All groups were sacrificed at 1 or 4 weeks after seizures. Animals were anesthetized with isoflurane and then perfused with $0.9 \%$ normal saline through the heart. Next, a brain sample was obtained by perfusion with $4 \%$ paraformaldehyde and fixed in the same paraformaldehyde fixative for $1 \mathrm{~h}$. One hour later, the brain was immersed in 30\% sucrose overnight. Usually, 1-2 days after a brain sample is taken, the sample sinks to the bottom of the sucrose solution. After the entire brain is frozen in the cryosection machine, the sample was cut into a $30-\mu \mathrm{m}$-thick cryostat at -20 to $-23^{\circ} \mathrm{C}$. The brain sections were stored at $-20{ }^{\circ} \mathrm{C}$ in a stock solution until histological evaluation.

\subsection{Detection of Live Neurons}

NeuN immunohistochemical staining to confirm live neuron was performed to evaluate whether a neuroprotective effect was obtained when apocynin was given after the pilocarpine-induced seizure. 
All immunostaining was performed on brain tissue sections (sliced at a $30 \mu \mathrm{m}$ thickness with a cryosection machine), and each of the five sections were analyzed. Monoclonal antibody-NeuN antibody (diluted 1:500, Billerica, Millipore Co., Burlington, MA, USA) was used as the primary antibody and incubated at $4{ }^{\circ} \mathrm{C}$. At the end of each step, each sample was washed three times with phosphate buffered saline (PBS) for $10 \mathrm{~min}$. Brain sections were diluted 1:250 with secondary antibody biotinylated anti-mouse immunoglobulin G (IgG, Vector, Burlingame, CA, USA) in the same manner as the primary antisera. After $2 \mathrm{~h}$ of incubation at room temperature (RT), tissues were washed and incubated with the ABC compound (Vector, Burlingame, CA, USA). Tissues were stained with the 3,3-diaminobenzidine (DAB, Sigma-Aldrich Co., St. Louis, MO, USA) color development method, and the sections were plated on slides [47].

\subsection{Detection of BrdU Labeling}

To determine the amount of newly generated cells and the effect of apocynin on the long-term survival and differentiation of these cells, BrdU was injected into the experimental group at $12 \mathrm{~h}$ intervals every day for 4 days. For immunoperoxidase detection of cells with BrdU incorporated, the sections were incubated with $0.3 \%$ hydrogen peroxide in methanol for $15 \mathrm{~min}$. The sections were incubated in $2 \mathrm{~N} \mathrm{HCl}$ for 90 min to denature the DNA and prepare for BrdU immunostaining, and then washed twice with $0.1 \mathrm{M}$ sodium borate buffer for $10 \mathrm{~min}$ to neutralize the acid. At the end of each step, each sample was washed three times with phosphate buffered saline (PBS) for $10 \mathrm{~min}$. In a solution containing $1 \%$ normal chicken serum and Triton X-100, a monoclonal antibody-BrdU antibody (diluted 1:150, Roche Co., Basel, Switzerland) was used as the primary antibody and incubated at room temperature for $1 \mathrm{~h}$. After attaching the primary antibody, the sections were incubated with secondary antibody (diluted 1:250 anti-mouse IgG) for $2 \mathrm{~h}$, at RT. Tissues were subjected to a RT reaction in a biotin-peroxidase complex (ABC, Vector Lab., Burlingame, CA, USA) for $2 \mathrm{~h}$, and then visualized using 3,3'-Diaminobenzidine (DAB, Sigma-Aldrich Co., St. Louis, MO, USA). Double immunofluorescence was used to confirm that the newly generated cells were differentiated into mature neurons. The sections were incubated with primary antibody for $2 \mathrm{~h}$, at RT, in mouse anti-NeuN (diluted 1:500, Billerica, Millipore Co., MA, USA) and rat anti-BrdU (diluted 1:150, Roche Co., Basel, Switzerland). Two hours later, the sections were washed three times with PBS and diluted with Alexa Fluor 488 donkey anti mouse IgG (NeuN) and Alexa Fluor 594 donkey anti-rat IgG (BrdU) secondary antibody (diluted 1:250, Invitrogen, Grand Island, NY, USA) incubated for $2 \mathrm{~h}$, at RT. BrdU and NeuN (Alexa 594 and 488) were used in the scanning mode using a Zeiss LSM 710 confocal imaging system (Carl Zeiss, Oberkochen, Germany) to detect fluorescent signals.

\subsection{Doublecortin (DCX) Immunohistochemistry}

To detect neuroblasts, brain slices were immunostained with guinea pig anti-doublecortin (DCX) antibody (1:1000, Santa Cruz Biotechnology, Santa Cruz, CA, USA) [48]. Brain sections were incubated overnight at $4{ }^{\circ} \mathrm{C}$ with primary DCX antibody. The sections were incubated with secondary antibody (diluted 1:250 guinea pig IgG) for $2 \mathrm{~h}$, at RT. Images of neuroblast were captured using an Olympus IX70 inverted microscope (Olympus Co., Tokyo and Shinjuku, Japan). The experimenter counted the number of neuroblasts in the subgranular zone (the SGZ in the DG) of both hippocampal lobes. The number of neruoblasts in the SGZ was quantified by the experimenter.

\subsection{HNE (4-Hydroxy-2-Nonenal) Immunohistochemistry}

Detection of oxidative damage in the hippocampal region of brain by $4 \mathrm{HNE}$ (4-hydroxy-2-nonenal) staining was evaluated by confirming lipid peroxidation products. 4HNE (Alpha Diagnostic International Inc., San Antonio, TX, USA) antibody immunostaining was performed as described above. The tissues were incubated overnight at $4{ }^{\circ} \mathrm{C}$ in PBS containing $0.3 \%$ Triton X-100 in a polyclonal rabbit anti-HNE antiserum compound (diluted 1:500, Alpha Diagnostic International Inc., San Antonio, TX, USA). After tissue incubation, brain tissue was incubated with Alexa Fluor 594 conjugated goat 
anti-rabbit IgG secondary antibody (Invitrogen, Grand Island, NY, USA) at a dilution of 1:250 for $2 \mathrm{~h}$, at RT. After secondary staining, the sections were washed three times for $10 \mathrm{~min}$ with PBS and plated on gelatin-coated slides. The stained tissue was observed for fluorescence using an Axioscope microscope (Carl Zeiss, Munchen Hallbergmoos, Germany) (Alexa 594) and the 4HNE intensity was quantified using image J (Laboratory for Optical and Computational Instrumentation (LOCI), University of Wisconsin, Madison, WI, USA).

\subsection{Data Analysis}

We compared each test group using repeated measures versus deviation analysis (ANOVA) and a Bonferroni post-test. Data were expressed as mean \pm standard error of the mean (S.E.M.) and were considered significant when $p<0.05$.

Author Contributions: S.H.L. researched the data, reviewed, and edited the manuscript. B.Y.C. reviewed and edited the manuscript. A.R.K., J.H.J., D.K.H., D.H.K., and B.S.K. researched the data. H.K.S. and H.C.C. performed the data analysis. S.W.S. contributed to the discussion and wrote, reviewed, and edited the manuscript. S.W.S. takes full responsibility for the manuscript and its originality. All authors read and approved the final manuscript.

Funding: This study was supported by funding from the National Research Foundation of Korea (NRF) (NRF-2017R1D1A1A09081190) to Sang Won Suh. This study was also supported by Hallym University Research Fund (HRF-201801-014).

Conflicts of Interest: The authors declare no conflict of interest.

\section{References}

1. Cavalheiro, E.A.; Leite, J.P.; Bortolotto, Z.A.; Turski, W.A.; Ikonomidou, C.; Turski, L. Long-term effects of pilocarpine in rats: Structural damage of the brain triggers kindling and spontaneous recurrent seizures. Epilepsia 1991, 32, 778-782. [CrossRef] [PubMed]

2. Curia, G.; Longo, D.; Biagini, G.; Jones, R.S.; Avoli, M. The pilocarpine model of temporal lobe epilepsy. J. Neurosci. Methods 2008, 172, 14-57. [CrossRef] [PubMed]

3. Navarro Mora, G.; Bramanti, P.; Osculati, F.; Chakir, A.; Nicolato, E.; Marzola, P.; Sbarbati, A.; Fabene, P.F. Does pilocarpine-induced epilepsy in adult rats require status epilepticus? PLoS ONE 2009, 4, e5759. [CrossRef] [PubMed]

4. Dayer, A.G.; Ford, A.A.; Cleaver, K.M.; Yassaee, M.; Cameron, H.A. Short-term and long-term survival of new neurons in the rat dentate gyrus. J. Comp. Neurol. 2003, 460, 563-572. [CrossRef] [PubMed]

5. Cameron, H.A.; Glover, L.R. Adult neurogenesis: Beyond learning and memory. Annu. Rev. Psychol. 2015, 66, 53-81. [CrossRef] [PubMed]

6. Feng, R.; Rampon, C.; Tang, Y.P.; Shrom, D.; Jin, J.; Kyin, M.; Sopher, B.; Miller, M.W.; Ware, C.B.; Martin, G.M.; et al. Deficient neurogenesis in forebrain-specific presenilin-1 knockout mice is associated with reduced clearance of hippocampal memory traces. Neuron 2001, 32, 911-926. [CrossRef]

7. Shors, T.J.; Miesegaes, G.; Beylin, A.; Zhao, M.; Rydel, T.; Gould, E. Neurogenesis in the adult is involved in the formation of trace memories. Nature 2001, 410, 372-376. [CrossRef] [PubMed]

8. Wasterlain, C.G.; Fujikawa, D.G.; Penix, L.; Sankar, R. Pathophysiological mechanisms of brain damage from status epilepticus. Epilepsia 1993, 34, S37-S53. [CrossRef] [PubMed]

9. Fujikawa, D.G.; Shinmei, S.S.; Cai, B. Seizure-induced neuronal necrosis: Implications for programmed cell death mechanisms. Epilepsia 2000, 41, S9-S13. [CrossRef] [PubMed]

10. Hattiangady, B.; Shetty, A.K. Implications of decreased hippocampal neurogenesis in chronic temporal lobe epilepsy. Epilepsia 2008, 49, 26-41. [CrossRef] [PubMed]

11. Dingledine, R.; Varvel, N.H.; Dudek, F.E. When and how do seizures kill neurons, and is cell death relevant to epileptogenesis? Adv. Exp. Med. Biol. 2014, 813, 109-122. [PubMed]

12. Parent, J.M.; Lowenstein, D.H. Seizure-induced neurogenesis: Are more new neurons good for an adult brain? Prog. Brain Res. 2002, 135, 121-131. [PubMed]

13. Rotheneichner, P.; Marschallinger, J.; Couillard-Despres, S.; Aigner, L. Neurogenesis and neuronal regeneration in status epilepticus. Epilepsia 2013, 54, 40-42. [CrossRef] [PubMed] 
14. Huang, T.T.; Zou, Y.; Corniola, R. Oxidative stress and adult neurogenesis-Effects of radiation and superoxide dismutase deficiency. Semin. Cell Dev. Biol. 2012, 23, 738-744. [CrossRef] [PubMed]

15. Hattiangady, B.; Rao, M.S.; Shetty, A.K. Chronic temporal lobe epilepsy is associated with severely declined dentate neurogenesis in the adult hippocampus. Neurobiol. Dis. 2004, 17, 473-490. [CrossRef] [PubMed]

16. Kralic, J.E.; Ledergerber, D.A.; Fritschy, J.M. Disruption of the neurogenic potential of the dentate gyrus in a mouse model of temporal lobe epilepsy with focal seizures. Eur. J. Neurosci. 2005, 22, 1916-1927. [CrossRef] [PubMed]

17. Li, N.; Zhang, G.; Yi, F.X.; Zou, A.P.; Li, P.L. Activation of NAD(P)H oxidase by outward movements of $\mathrm{H}^{+}$ ions in renal medullary thick ascending limb of Henle. Am. J. Physiol. Ren. Physiol. 2005, 289, F1048-1056. [CrossRef] [PubMed]

18. Vejrazka, M.; Micek, R.; Stipek, S. Apocynin inhibits NADPH oxidase in phagocytes but stimulates ROS production in non-phagocytic cells. Biochim. Biophys. Acta 2005, 1722, 143-147. [CrossRef] [PubMed]

19. Groemping, Y.; Rittinger, K. Activation and assembly of the NADPH oxidase: A structural perspective. Biochem. J. 2005, 386, 401-416. [CrossRef] [PubMed]

20. Brennan, A.M.; Suh, S.W.; Won, S.J.; Narasimhan, P.; Kauppinen, T.M.; Lee, H.; Edling, Y.; Chan, P.H.; Swanson, R.A. NADPH oxidase is the primary source of superoxide induced by NMDA receptor activation. Nat. Neurosci. 2009, 12, 857-863. [CrossRef] [PubMed]

21. Kim, J.H.; Jang, B.G.; Choi, B.Y.; Kim, H.S.; Sohn, M.; Chung, T.N.; Choi, H.C.; Song, H.K.; Suh, S.W. Post-treatment of an NADPH oxidase inhibitor prevents seizure-induced neuronal death. Brain Res. 2013, 1499, 163-172. [CrossRef] [PubMed]

22. Choi, B.Y.; Jang, B.G.; Kim, J.H.; Lee, B.E.; Sohn, M.; Song, H.K.; Suh, S.W. Prevention of traumatic brain injury-induced neuronal death by inhibition of NADPH oxidase activation. Brain Res. 2012, 1481, 49-58. [CrossRef] [PubMed]

23. Choi, B.Y.; Kim, J.H.; Kho, A.R.; Kim, I.Y.; Lee, S.H.; Lee, B.E.; Choi, E.; Sohn, M.; Stevenson, M.; Chung, T.N.; et al. Inhibition of NADPH oxidase activation reduces EAE-induced white matter damage in mice. J. Neuroinflamm. 2015, 12, 104. [CrossRef] [PubMed]

24. Pestana, R.R.; Kinjo, E.R.; Hernandes, M.S.; Britto, L.R. Reactive oxygen species generated by NADPH oxidase are involved in neurodegeneration in the pilocarpine model of temporal lobe epilepsy. Neurosci. Lett. 2010, 484, 187-191. [CrossRef] [PubMed]

25. Simonyi, A.; Serfozo, P.; Lehmidi, T.M.; Cui, J.; Gu, Z.; Lubahn, D.B.; Sun, A.Y.; Sun, G.Y. The neuroprotective effects of apocynin. Front. Biosci. 2012, 4, 2183-2193. [CrossRef]

26. Suh, S.W.; Gum, E.T.; Hamby, A.M.; Chan, P.H.; Swanson, R.A. Hypoglycemic neuronal death is triggered by glucose reperfusion and activation of neuronal NADPH oxidase. J. Clin. Investig. 2007, 117, 910-918. [CrossRef] [PubMed]

27. Suh, S.W.; Hamby, A.M.; Gum, E.T.; Shin, B.S.; Won, S.J.; Sheline, C.T.; Chan, P.H.; Swanson, R.A. Sequential release of nitric oxide, zinc, and superoxide in hypoglycemic neuronal death. J. Cereb. Blood Flow Metab. 2008, 28, 1697-1706. [CrossRef] [PubMed]

28. Suh, S.W.; Shin, B.S.; Ma, H.; Van Hoecke, M.; Brennan, A.M.; Yenari, M.A.; Swanson, R.A. Glucose and NADPH oxidase drive neuronal superoxide formation in stroke. Ann. Neurol. 2008, 64, 654-663. [CrossRef] [PubMed]

29. Hakami, N.Y.; Ranjan, A.K.; Hardikar, A.A.; Dusting, G.J.; Peshavariya, H.M. Role of NADPH Oxidase-4 in Human Endothelial Progenitor Cells. Front. Physiol. 2017, 8, 150. [CrossRef] [PubMed]

30. Wang, J.; Ma, M.W.; Dhandapani, K.M.; Brann, D.W. Regulatory role of NADPH oxidase 2 in the polarization dynamics and neurotoxicity of microglia/macrophages after traumatic brain injury. Free Radic. Biol. Med. 2017, 113, 119-131. [CrossRef] [PubMed]

31. Wang, J.; Ma, M.W.; Dhandapani, K.M.; Brann, D.W. NADPH oxidase 2 deletion enhances neurogenesis following traumatic brain injury. Free Radic. Biol. Med. 2018, 123, 62-71. [CrossRef] [PubMed]

32. French, J.A.; Williamson, P.D.; Thadani, V.M.; Darcey, T.M.; Mattson, R.H.; Spencer, S.S.; Spencer, D.D. Characteristics of medial temporal lobe epilepsy: I. Results of history and physical examination. Ann. Neurol. 1993, 34, 774-780. [CrossRef] [PubMed] 
33. Russo, R.; Cattaneo, F.; Lippiello, P.; Cristiano, C.; Zurlo, F.; Castaldo, M.; Irace, C.; Borsello, T.; Santamaria, R.; Ammendola, R.; et al. Motor coordination and synaptic plasticity deficits are associated with increased cerebellar activity of NADPH oxidase, CAMKII, and PKC at preplaque stage in the TgCRND8 mouse model of Alzheimer's disease. Neurobiol. Aging 2018, 68, 123-133. [CrossRef] [PubMed]

34. Freitas, R.M.; Vasconcelos, S.M.; Souza, F.C.; Viana, G.S.; Fonteles, M.M. Oxidative stress in the hippocampus after pilocarpine-induced status epilepticus in Wistar rats. FEBS J. 2005, 272, 1307-1312. [CrossRef] [PubMed]

35. Bedard, K.; Krause, K.H. The NOX family of ROS-generating NADPH oxidases: Physiology and pathophysiology. Physiol. Rev. 2007, 87, 245-313. [CrossRef] [PubMed]

36. Klein, J.A.; Ackerman, S.L. Oxidative stress, cell cycle, and neurodegeneration. J. Clin. Investig. 2003, 111, 785-793. [CrossRef] [PubMed]

37. Liu, J.; Wang, A.; Li, L.; Huang, Y.; Xue, P.; Hao, A. Oxidative stress mediates hippocampal neuron death in rats after lithium-pilocarpine-induced status epilepticus. Seizure 2010, 19, 165-172. [CrossRef] [PubMed]

38. Cattaneo, F.; Guerra, G.; Parisi, M.; De Marinis, M.; Tafuri, D.; Cinelli, M.; Ammendola, R. Cell-surface receptors transactivation mediated by g protein-coupled receptors. Int. J. Mol. Sci. 2014, 15, 19700-19728. [CrossRef] [PubMed]

39. Michiels, C.; Raes, M.; Toussaint, O.; Remacle, J. Importance of Se-glutathione peroxidase, catalase, and $\mathrm{Cu} / \mathrm{Zn}-\mathrm{SOD}$ for cell survival against oxidative stress. Free Radic. Biol. Med. 1994, 17, 235-248. [CrossRef]

40. Kuhn, H.G.; Dickinson-Anson, H.; Gage, F.H. Neurogenesis in the dentate gyrus of the adult rat: Age-related decrease of neuronal progenitor proliferation. J. Neurosci. 1996, 16, 2027-2033. [CrossRef] [PubMed]

41. Seki, T.; Arai, Y. Highly polysialylated neural cell adhesion molecule (NCAM-H) is expressed by newly generated granule cells in the dentate gyrus of the adult rat. J. Neurosci. 1993, 13, 2351-2358. [CrossRef] [PubMed]

42. Cameron, H.A.; Woolley, C.S.; McEwen, B.S.; Gould, E. Differentiation of newly born neurons and glia in the dentate gyrus of the adult rat. Neuroscience 1993, 56, 337-344. [CrossRef]

43. van Rijckevorsel, K. Cognitive problems related to epilepsy syndromes, especially malignant epilepsies. Seizure 2006, 15, 227-234. [CrossRef] [PubMed]

44. Persinger, M.A.; Makarec, K.; Bradley, J.C. Characteristics of limbic seizures evoked by peripheral injections of lithium and pilocarpine. Physiol. Behav. 1988, 44, 27-37. [CrossRef]

45. Racine, R.J.; Gartner, J.G.; Burnham, W.M. Epileptiform activity and neural plasticity in limbic structures. Brain Res. 1972, 47, 262-268. [CrossRef]

46. Kim, J.E.; Kim, Y.J.; Kim, J.Y.; Kang, T.C. PARP1 activation/expression modulates regional-specific neuronal and glial responses to seizure in a hemodynamic-independent manner. Cell Death Dis. 2014, 5, e1362. [CrossRef] [PubMed]

47. Choi, B.Y.; Kim, J.H.; Kim, H.J.; Lee, B.E.; Kim, I.Y.; Sohn, M.; Suh, S.W. Zinc chelation reduces traumatic brain injury-induced neurogenesis in the subgranular zone of the hippocampal dentate gyrus. J. Trace Elem. Med. Biol. 2014, 28, 474-481. [CrossRef] [PubMed]

48. Kim, J.H.; Jang, B.G.; Choi, B.Y.; Kwon, L.M.; Sohn, M.; Song, H.K.; Suh, S.W. Zinc chelation reduces hippocampal neurogenesis after pilocarpine-induced seizure. PLoS ONE 2012, 7, e48543. [CrossRef] [PubMed]

(C) 2018 by the authors. Licensee MDPI, Basel, Switzerland. This article is an open access article distributed under the terms and conditions of the Creative Commons Attribution (CC BY) license (http://creativecommons.org/licenses/by/4.0/). 